\title{
Fatores predisponentes para a ocorrência de complicações locais da terapia intravenosa periférica em crianças/adolescentes com câncer.
}

\author{
Elis Souza Machado' ${ }^{1,}$ Luciano Marques dos Santos ${ }^{2}$ \\ ${ }^{1}$ Bolsista PEVIC/CNPq, Graduanda em Enfermagem, Universidade Estadual de Feira de Santana, e- \\ mail:elis.uefs@hotmail.com
}

${ }^{2}$ Orientador. Mestre em Enfermagem. Professor Auxiliar do Curso de Graduação em Enfermagem da UEFS. Coordenador do Projeto de Pesquisa "Segurança do paciente pediátrico e sua família: estudo das tecnologias e eventos adversos relacionados à terapia intravascular periférica". E-mail: lucmarxenfo@ yahoo.com.br.

Palavras chave: Enfermagem Oncológica. Eventos adversos. Cateterismo venoso periférico.

\section{INTRODUÇÃO}

O câncer infantil corresponde a um grupo de várias doenças que têm em comum a proliferação descontrolada de células anormais e que pode ocorrer em qualquer local do organismo. Este tipo de câncer era considerado uma doença aguda com mau prognóstico, porém atualmente, apresenta grande possibilidade de cura, com potencial aumento de sobrevida em aproximadamente $70 \%$ dos casos. Entretanto, esse tratamento ainda com grande potencial de cura é acompanhado por dor e sofrimento para a criança, principalmente relacionado aos inúmeros procedimentos invasivos a que as mesmas são submetidas.

Dentre as diversas opções de tratamento oncológico, o mais utilizado e o mais invasivo desses é a quimioterapia. A administração de quimioterápicos por via intravenosa em ciclos repetidos é um dos pilares do tratamento de pacientes com câncer(VILLARÍN; ARAÚJO, 2003). Entretanto, essa modalidade de tratamento resulta em certas complicações como flebites, infiltrações e extravasamento. Villarín e Belda (2014) afirmam que o extravasamento é a complicação da quimioterapia mais grave que pode ocorrer em doentes que recebe este tratamento, devido ao potencial nocivo de uma parte destas drogas,resultando em bolhas ou efeito necrosante em tecidos que se infiltram.

Por isso, o manuseio e a administração intravenosa de quimioterápicos para o tratamento de pacientes com câncer é uma técnica que, dada a sua complexidade, requer uma equipe qualificada, conhecedora de seus efeitos adversos e potencial de riscos(VILLARÍN; BELDA, 2014). Assim o objetivo da pesquisa é a investigação dos fatores predisponentes para a ocorrência de complicações locais da terapia intravenosa periférica (TIV) em crianças/adolescentes com câncer, de forma ajudar no conhecimento sobre a temática para auxilio na qualificação dos profissionais da área, já que durante a busca pelo referencial teórico para a produção da pesquisa foi constatada aincipiente produção do conhecimento sobre esta temática.

\section{METODOLOGIA}


Trata-se de um estudo transversal. E para a confecção do mesmo, foram utilizados dados secundários do projeto de pesquisa "Segurança do paciente pediátrico e sua família: estudo de tecnologias e eventos adversos relacionados à terapia intravascular periférica" que foi aprovado pelo Comitê de Ética na Pesquisa (CEP) da UEFS nº 841.612. Foi consultado o banco de dados do referido projeto e selecionada as informações das crianças/adolescentescom câncer e que foram submetidas à cateterização intravenosa periférica (CIP) nas unidades de ambulatório de oncologia ou clínica oncológica do Hospital Estadual da Criança (HEC).

A coleta dos dados ocorreuatravés de consulta á fonte primária, mediante observação da CIP em cada criança/adolescente selecionado e do prontuário destes pelos pesquisadores. Além de ser utilizado também um formulário, contendo dados sócio demográficos e clínicos do participante do estudo, características da CIP,condições da rede venosa acessada, TIV prévia e atual, além do motivo para a retirada do cateter.Estes acessos vasculares periféricos são acompanhados do início da CIP até o momento da retirada em decorrência de alta hospitalar, término da TIV ou ocorrência de complicação.

\section{RESULTADOS E DISCUSSÃO}

Durante o período do estudo, foram acompanhadas um total de 333 punções na unidade oncológica, ocorrendo 62 complicações. Dentre essas, a mais frequente foi o extravasamento, representando 41,9\% das complicações, seguidas de: flebite com $24,2 \%$, infiltração $19,4 \%$ e com menor porcentagem a obstrução representando 14,5\% dos casos (tabela 1). Na ocorrência da complicação flebite ( $2^{\mathrm{a}}$ mais presente), o grau mais frequente foi o 1 , assim como analisando a complicação infiltração, tem-se que o grau mais frequente foi o 4, classificado como um extravasamento.

TABELA 1: Prevalência de complicações da TIV segundo CIP realizadas em uma unidade de clínica oncológica pediátrica no interior da Bahia. Feira de Santana, abril 2015 - dezembro 2016.

\begin{tabular}{lcc}
\hline \multicolumn{1}{c}{ Variáveis } & Clínica Oncológica & \\
\hline Ocorrência de Complicações & $\mathbf{N}$ & $\%$ \\
Sim & 62 & $18,6 \%$ \\
Não & 271 & $81,4 \%$ \\
Tipo de Complicação & & \\
Flebite & 15 & $24,2 \%$ \\
Extravasamento & 26 & $41,9 \%$ \\
Infiltração & 12 & $19,4 \%$ \\
Obstrucão & 9 & $14,5 \%$ \\
\hline
\end{tabular}

FONTE: Coleta de dados, Feira de Santana/Bahia, 2016. 
Após as modelagens realizadas, pôde-se confirmar quais os fatores que agiam como predisponentes para a ocorrência das complicações em crianças/adolescentes com câncer. Esses fatores estavam relacionados àsvariáveis da TIV prévia e atual. Constatou-se assim que o uso da TIV periférica prolongada $(\mathrm{p}=0,002)$ e o antecedente para complicação $(\mathrm{p}<0,001)$ eram fatores de predisposição para a ocorrência de complicações da TIV.

TABELA 2: Regressão de Poisson das variáveis relacionadas à TIV prévia associadas à ocorrência de complicação na unidade de clínica oncológica no interior da Bahia. Feira de Santana, abril 2015 - dezembro 2016

\begin{tabular}{lccc}
\hline \multicolumn{4}{c}{ Complicações da terapia intravenosa } \\
\hline \multicolumn{1}{c}{ Variáveis } & RP & IC & p-valor \\
\hline TIV periférica prolongada & 3,44 & $1,58-7,50$ & 0,002 \\
Antecedente de complicações & 4,22 & $2,84-6,26$ & $<0,001$ \\
\hline
\end{tabular}

FONTE: Coleta de dados, Feira de Santana/Bahia, 2016.

O modelo das variáveis relacionadas à TIV atual confirmou que as utilizações de medicamentos vesicantes $(\mathrm{p}=0,006)$ e soluções vesicantes $(\mathrm{p}<0,001)$ foram fatores associados á ocorrência de complicações nessas crianças/adolescentes (tabela 2).

TABELA 3: Regressão de Poisson das variáveis relacionadas à TIV atual associadas à ocorrência de complicação na unidade de clínica oncológica no interior da Bahia. Feira de Santana, abril 2015 - dezembro 2016

\begin{tabular}{lccc}
\hline \multicolumn{4}{c}{ Complicações da terapia intravenosa } \\
\hline \multicolumn{1}{c}{ Variáveis } & RP & IC & p-valor \\
\hline Utilização de medicamentos vesicantes & 0,54 & $0,36-0,84$ & 0,006 \\
Utilização de solução vesicante & 2,65 & $1,69-4,17$ & $<0,001$ \\
\hline
\end{tabular}

FONTE: Coleta de dados, Feira de Santana/Bahia, 2016.

Analisando as complicações e os graus, o extravasamento apresentou uma frequência de 41,9\% dos casos, sendo esta a complicação mais frequente. Porém segundo XAVIER et al., 2011, toda infiltração deve ser classificada como uma infiltração, desta forma verifica-se que a complicação mais frequente foi a infiltração. Esse resultado converge com parâmetros encontrados em estudos que indicam que acomplicação mais frequente em crianças é a infiltração, com 72\% dos casos (ABUSAFIA; BOZTEPE, 2017).

Com relação à TIV prévia, as crianças/adolescentes apresentaram 3 grandes variáveis com associações estatisticamente significantes, foram elas: histórico de dificuldade para a CIP, antecedentes de complicações e TIV periférica prolongada. Para o histórico de dificuldade para CIP, a prevalência sobre as crianças/adolescentes sem essa dificuldade foi 1,619 vezes maior. Ainda com essa significância estatística Passos (2016) aponta que essa variável não estava associada à ocorrência de flebite em crianças/adolescentes com câncer.

Quanto as variáveis relacionadas à TIV atual pode-se inferir que as crianças/adolescentes que fazem uso de medicamentos vesicantes e soluções vesicantes, apresentaram prevalência maior para a ocorrência de complicações, se comparadas as que não utilizaram os 
medicamentos e as soluções com essas naturezas.Entretanto as que utilizaram medicamentos vesicantes dispuseram de prevalência 0,49 vezes menor para a ocorrência de complicações. Os resultados dos estudos de Jacinto, Avelar e Pedreira (2011) e Jacinto et al. (2014) concordaram com os achados dessa pesquisa, pois a utilização de soluções ou medicamentos com potencial risco (osmolaridade superior a $350 \mathrm{mOsm} / \mathrm{L}$, $\mathrm{pH}$ menor que 5 ou maior que 9) predispuseram a ocorrência de infiltração e flebite, respectivamente.

\section{CONCLUSÃO}

Este estudo foi capaz assim, de alcançar os objetivos que haviam sidos propostos, já que foi possível verificar alguns dos fatores predisponentes para a ocorrência de complicações decorrentes da TIV por via periférica em crianças/adolescentes com câncer. A regressão de Poisson apresentou a seguinte modelagem das variáveis relacionadas às crianças/adolescentes: TIV periférica prolongada $(\mathrm{p}=0,002)$, antecedentes de complicações ( $p=0,000)$, utilizações de medicamentos vesicantes $(p=0,006)$ e soluções vesicantes $(\mathrm{p}=0,000)$.

Acredita-se que com esse estudo e as analises provenientes deste, sejam implantadas melhorias que abracem todos os envolvidos no processo da terapia intravenosa. A equipe de enfermagem, para que possa desenvolver ou aprimorar as técnicas existentes como forma de identificar precocemente uma complicação e assim á evite ou á trate, e a criança de forma que essa sofra o menos com procedimentos recorrentemente invasivos que já causam dor, e ainda sofram com complicações advindas desses procedimentos como a punção, que é tão importante para o tratamento quimioterápico.

\section{REFERÊNCIAS}

ABUSAFIA, B. M. R.; BOZTEPE, H. Evaluation of peripheral intravenous catheter-induced local complications in pediatrics.Accepted Article, 2017.

AVELAR, A. F. M.; PETERLINI, M. A. S.; PEDREIRA, M. L. G. Assertividade e tempo de permanência de cateteres intravenosos periféricos com inserção guiada por ultrassonografia em crianças e adolescentes. Rev. esc. enferm. USP, v. 47, n. 3, p. 539-546, 2013.

JACINTO, A. K. L. et al. Flebite associada a cateteres intravenosos periféricos em crianças: estudo de fatores predisponentes. Esc Anna Nery, v. 18, n. 2, p. 220-226. 2014.

PASSOS, F. S. Condições associadas à ocorrência de flebite em cateteres vasculares periféricos em crianças e adolescentes com câncer. 2016. 76f. Monografia (Graduação em Enfermagem) - Universidade Estadual de Feira de Santana, Feira de Santana, 2016. 